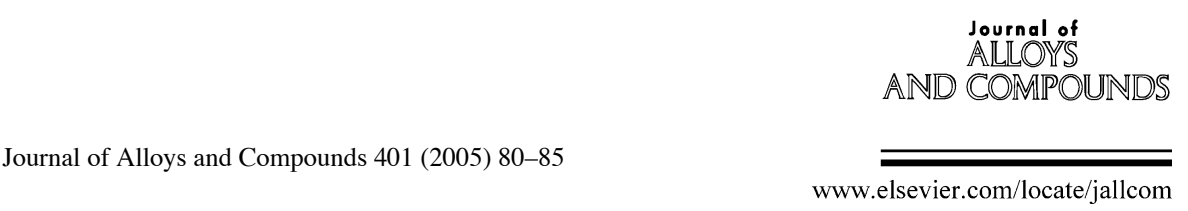

\title{
Spectroscopic infrared scanning near-field optical microscopy (IR-SNOM)
}

\author{
D. Vobornik $^{\mathrm{a}, *}$, G. Margaritondo ${ }^{\mathrm{a}}$, J.S. Sanghera ${ }^{\mathrm{b}}$,

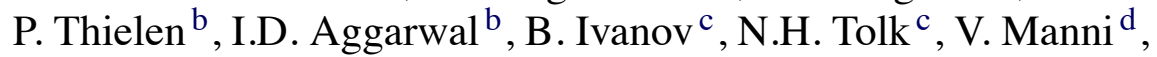 \\ S. Grimaldi ${ }^{\mathrm{d}}$, A. Lisi ${ }^{\mathrm{d}}$, S. Rieti $^{\mathrm{d}}{ }^{\text {, D.W. Piston }}{ }^{\mathrm{e}}$, \\ R. Generosi ${ }^{\text {f }}$, M. Luce $^{f}$, P. Perfetti ${ }^{\text {f }}$, A. Cricenti ${ }^{f}$ \\ a Institut de Physique de la Matière Complexe, Ecole Polytechnique Fédérale de Lausanne (EPFL), Station 3, CH-1015 Lausanne, Switzerland \\ b Optical Sciences Division, U.S. Naval Research Laboratory, 4555 Overlook Avenue SE, Washington, DC 20375, USA \\ ${ }^{\mathrm{c}}$ Department of Physics and Astronomy, Vanderbilt University, Nashville, TN 31235, USA \\ $\mathrm{d}$ Institute of Neurobiology and Molecular Medicine, 00133 Rome, Italy \\ e Department of Molecular Physiology and Biophysics, Vanderbilt University, Nashville, TN 37232, USA \\ ${ }^{\mathrm{f}}$ Istituto di Stuttura della Materia, via Fosso del Cavaliere 100, 00133 Rome, Italy \\ Received 9 September 2004; received in revised form 14 January 2005; accepted 3 February 2005
}

\begin{abstract}
Scanning near-field optical microscopy (SNOM or NSOM) is the technique with the highest lateral optical resolution available today, while infrared (IR) spectroscopy has a high chemical specificity. Combining SNOM with a tunable IR source produces a unique tool, IR-SNOM, capable of imaging distributions of chemical species with a $100 \mathrm{~nm}$ spatial resolution. We present in this paper boron nitride (BN) thin film images, where IR-SNOM shows the distribution of hexagonal and cubic phases within the sample. Exciting potential applications in biophysics and medical sciences are illustrated with SNOM images of the distribution of different chemical species within cells. We present in this article images with resolutions of the order of $\lambda / 60$ with SNOM working with infrared light. With our SNOM setup, we routinely get optical resolutions between 50 and $150 \mathrm{~nm}$, regardless of the wavelength of the light used to illuminate the sample.
\end{abstract}

(C) 2005 Elsevier B.V. All rights reserved.

Keywords: SNOM; NSOM; Infrared spectroscopy; BN; Cells; Thin film

\section{Introduction}

In the 1870s, Ernst Abbe demonstrated, closely followed by Lord Rayleigh, that, due to the diffraction, the lens-based microscopes are limited in their resolution to no better than $\lambda / 2 n \sin \theta$ ( $\lambda$ being the wavelength of the light used to illuminate the object, $n$ the refractive index of the medium, and $\theta$ the aperture half-angle of the optical system) [1-4]. Therefore, the best resolution achievable using visible light with conventional microscopes is around $300 \mathrm{~nm}$. The diffraction limit is even

\footnotetext{
* Corresponding author. Tel.: +41 2169354 22; fax: +41 216934666.

E-mail address: dusan.vobornik@epfl.ch (D. Vobornik).
}

more troublesome with the relatively long wavelengths of the infrared light, limiting the resolution to several microns.

Many microscopy techniques (scanning tunneling microscopy (STM), atomic force microscopy (AFM), scanning electron microscopy (SEM), etc.) were developed in order to overcome this limit. Still, none of these microscopy techniques is capable of gathering optical information about the sample. SNOM is a purely optical microscopy technique, which allows us to form images with resolution well beyond the diffraction limit [3-9]. We will present in this article images with resolutions of the order of $\lambda / 60$ with SNOM working with infrared light. With our SNOM setup, we routinely get optical resolutions between 50 and $150 \mathrm{~nm}$, 
regardless of the wavelength of the light used to illuminate the sample [4,10-12].

\section{SNOM fundamentals}

With a conventional microscope (Fig. 1, on the left), we actually detect a superposition of light coming from every part of the sample under the objective, which means a superposition of diffraction patterns of every shape on the sample. When the distance between the two shapes becomes smaller than $\lambda / 2 n \sin \theta$ we can not resolve the two objects anymore $[1,2]$.

SNOM collects light through a small aperture at the tip of a tapered optical fiber that is scanned over the sample (Fig. 1, on the right). The light is conducted from the sample, through the fiber, to the detector. In our experiments, the sample was illuminated from the outside, as shown on the right side of Fig. 1. During the scanning, the fiber tip is at a constant and a very small distance (of the order of few nanometers) from the sample. The signal is collected only from a very small portion of the sample, approximately the same size as the aperture at the optical fiber tip. The resolution is no longer limited by the diffraction; it depends solely on the size of the aperture of the optical fiber [3-8].

It is worth mentioning that there is also a so-called apertureless SNOM. The apertureless SNOM is not based on an optical fiber, but on a sharp metallic tip that scans the sample. Because the technology enables the fabrication of metallic tips that are much sharper (atomic size STM tips, for example) than the optical fiber tips $(\sim 50 \mathrm{~nm}$ diameter is the maximum at the moment), this technique can potentially achieve much better resolutions. Some interesting apertureless IR-SNOM results have been reported $[13,14]$.

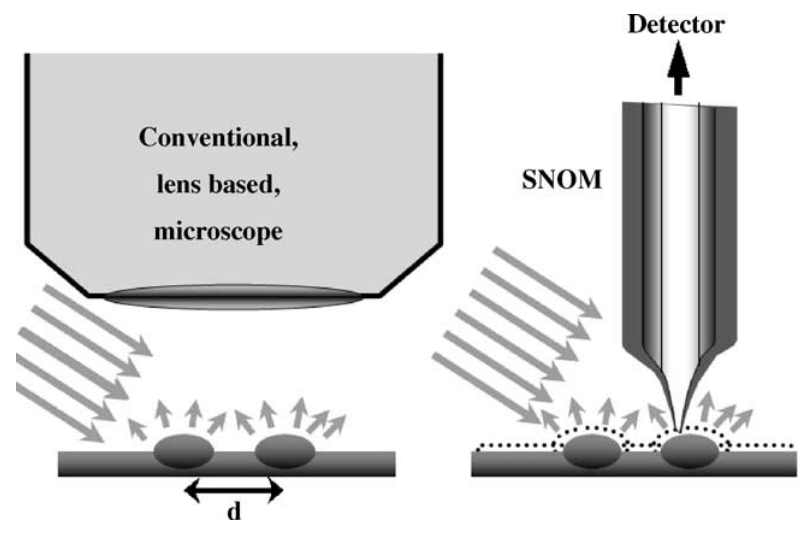

Fig. 1. Conventional optical microscope (left) collects the light from all over the sample; image is a superposition of diffraction patterns of every shape on the sample. SNOM optical fiber tip (right) scans the sample, acquires the total reflected intensity from each scan point and integrates it as one pixel into the final image. SNOM resolution is no longer limited by the diffraction; it depends solely on the size of the aperture of the optical fiber.

\section{Experimental details}

There were important experimental challenges to overcome in order to perform successful spectroscopic IR-SNOM experiments. For example, all the aperture SNOM setups working with visible light use the telecommunications' silica optical fibers. These fibers have strong absorption in the IR spectral region and are therefore unsuitable for IR-SNOM. We also needed a tunable, monochromatic IR source in order to perform spectroscopic measurements.

The fabrication of extremely high quality infrared fiber tips is a crucial technical step in the practical realization of our experiments. Tapered IR SNOM probes are obtained from single-mode, $1 \mathrm{~m}$ long arsenic selenide fibers with a $70 \mu \mathrm{m}$ clad thickness and a $120 \mu \mathrm{m}$ core diameter (Fig. 2) [4,15-17]. One end of the fiber is interfaced to a InSb detector. The other end of the fiber is chemically etched using a protective layer etching system. The etching solution was 7:3 $\left(\mathrm{H}_{2} \mathrm{O}_{2}: \mathrm{H}_{2} \mathrm{SO}_{4}\right)$. An optical microscope is used to monitor the etching process. The etched tips are coated with gold using a Varian thermal deposition system at a pressure of $10^{-6}$ Torr with the tips at a $25^{\circ}-30^{\circ}$ angle above the evaporation point source to create an aperture. The tips are rotated to achieve a uniform coating with a thickness of 100-125 nm [4,15-17].

Another key technical problem in IR spectroscopic SNOM is the need for the IR source to be tunable over a broad band and intense enough to offset the limited sub-wavelength aperture transmission. The Vanderbilt University free electron laser (FEL) is continuously tunable over a broad wavelength range $(2.1-9.8 \mu \mathrm{m})$ and is intense and stable, therefore, ideal for IR spectroscopic SNOM. While the use of a free electron laser source limits applications to the relatively few researchers who can access such a device, in principle the general technique could be applied to alternative tunable IR laser sources. Recently interesting IR-SNOM measurements have been done using a color center laser pumped by a $\mathrm{Kr}^{+}$ laser [18,19].

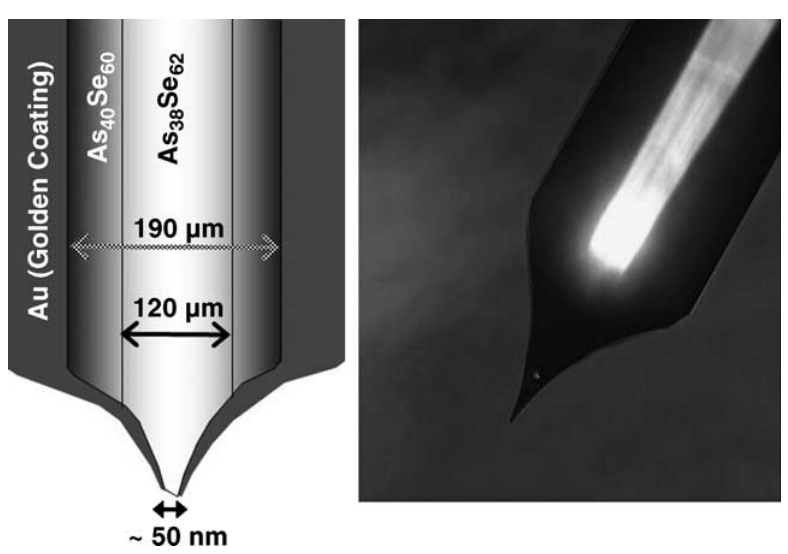

Fig. 2. Right side: tapered end of the chalcogenide optical fiber under a microscope. Left side: cross-section of the fiber with its main characteristics. 
We use the shear force feedback mechanism to control the tip-to-surface distance. Resonant $(\sim 5 \mathrm{KHz})$ lateral $(x-y$ plane) oscillation of the fiber is induced. When the fiber's tip is at a few nanometers from the sample, the amplitude of the oscillation starts to be damped by shear forces (typically Van der Waals forces). The amplitude is monitored and fed into a feedback loop, which then moves the tip in the $z$ direction (towards and from the sample) in order to always have a constant damped amplitude of the oscillation (typically, we get the best results with 10-20\% damping). SNOM actually gives two images since the topography, similar to that given by the AFM, is acquired simultaneously with the optical image.

The FEL beam illuminates the specimen over a broad area ( $\sim 1 \mathrm{~mm}$ spot diameter) and the SNOM probe collects the reflected light.

\section{Boron nitride films results}

Cubic boron nitride (c-BN) films have physical and chemical properties very similar to those of diamond: extreme hardness, excellent chemical inertness, thermal stability and wide band gap. Cubic boron nitride is superior to diamond in some aspects. For example, diamond is readily oxidized at elevated temperature while c-BN is not. Recently, this stimulated an important amount of research on c-BN films synthesis. However, the growth of c-BN is difficult: the typical crystal phase of BN films is a mixture of the cubic and of the hexagonal (h-BN) phase [20], which is less attractive for industrial use.

In our experiments, BN films are grown on silicon wafers by laser induced chemical vapor deposition (LCVD). Undesired hexagonal and wurtzite crystal phases are always
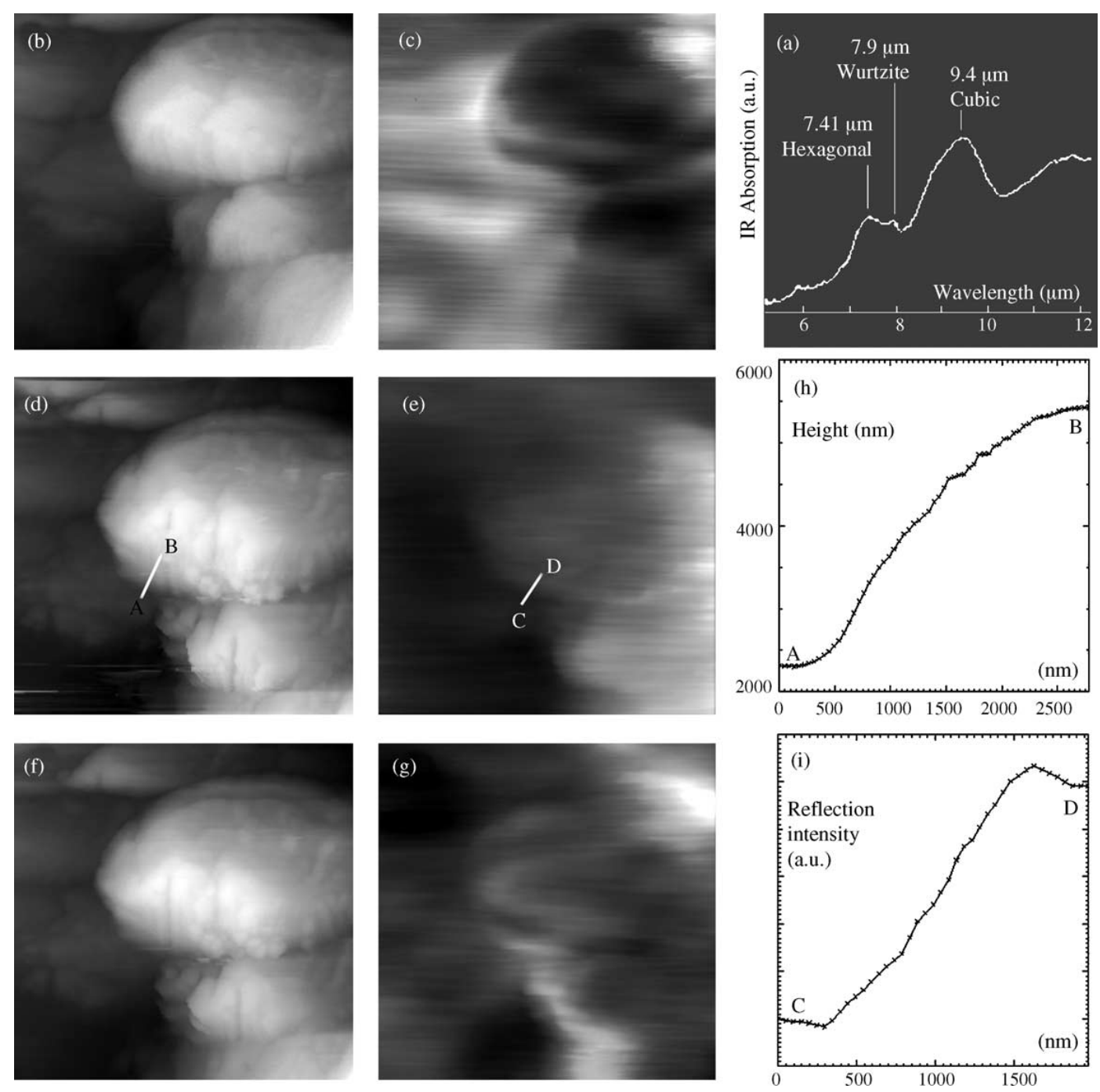

Fig. 3. BN results: (a) FT-IR spectrum with the vibrational modes corresponding to three crystallographic phases; (c), (e) and (g) are spectroscopic (reflectivity) SNOM images at the wavelengths 9.4, 7.9 and $7.41 \mu \mathrm{m}$; (b), (d) and (f) are corresponding topographic images; (h) is a topography cross-section corresponding to the line A-B in (d) and (g) is an intensity profile for the line C-D in (e). 

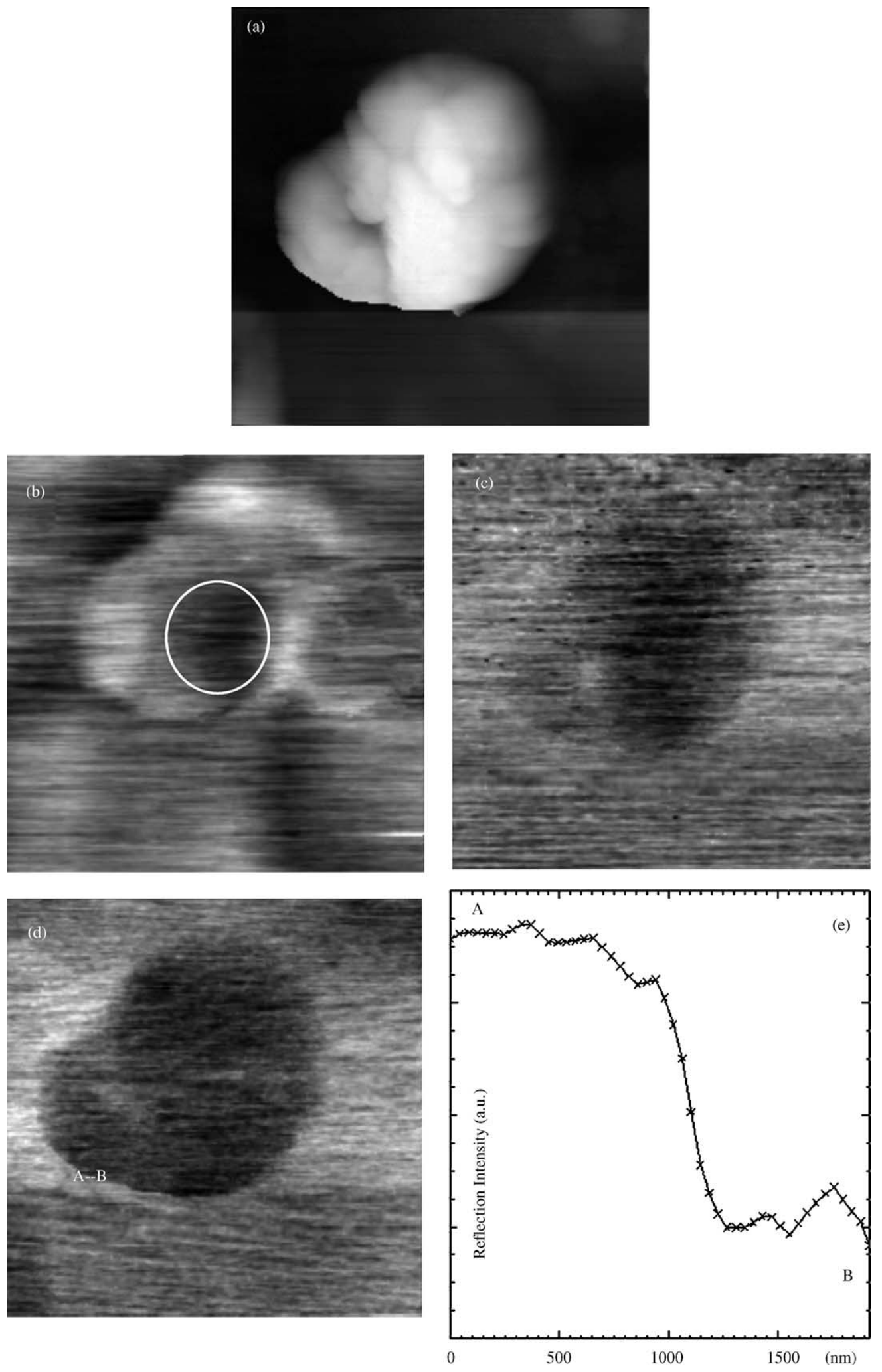

Fig. 4. Cell SNOM images: (a) shows the topography; (b), (c) and (d) are reflectivity SNOM images at the wavelengths $8.05,6.95$ and $6.1 \mu \mathrm{m}$, respectively; (e) shows a profile of the reflected intensity along the line A-B (in (d)), demonstrating the resolution of the order of $100 \mathrm{~nm}$ (or $\lambda / 60,30$ times superior to the resolution of the conventional lens-based microscopes). 
present in the films. Our experiment targets different vibration modes corresponding to these different crystallographic phases present in the BN film.

The FT-IR spectrum of Fig. 3a reveals indeed several vibration modes. Of these, the features at 9.4, 7.9 and $7.4 \mu \mathrm{m}$ are associated respectively to the cubic, wurtzite and hexagonal BN crystal phases. The spectroscopic (reflectivity) SNOM images of Fig. 3c (acquired with FEL illuminating the sample with $\lambda=9.42 \mu \mathrm{m}$, strongly absorbed by c-BN), Fig. 3e (acquired with FEL illuminating the sample with $\lambda=7.94 \mu \mathrm{m}$, strongly absorbed by w-BN) and Fig. $3 \mathrm{~g}$ (acquired with FEL illuminating the sample with $\lambda=7.41 \mu \mathrm{m}$, strongly absorbed by h-BN) clearly reveal differences in the lateral distribution of such phases. Darker color on these images indicates more absorption.

With our setup each scan gives simultaneously two images: the topographic and the optical one. Fig. 3b, d and $f$ are, respectively, topographic images corresponding to Fig. 3c, e and g. They show that the three scans have been performed over the same part of the sample.

The strongest absorption from the cubic phase (Fig. 3c) arises from the three large grains that can be seen on the right side of the topographic images. In contrast, the wurtzite phase (Fig. 3e) is mainly present on the left side of the image, in the lower parts of the sample, closer to the substrate. A weak hexagonal contribution (Fig. $3 \mathrm{~g}$ ) seems to be coming from all over the sample. The lower intensity of the signal can be explained if we suppose that hexagonal phase is actually on the interface with the substrate, under the layer of the c-BN. Our results confirm similar observations reported with other experimental techniques [20]. However, SNOM offers several advantages over the experimental technique used in [20]: it is completely non-destructive and provides additional information, such as the size of the grains for example.

Fig. 3i shows a reflected signal cross-section along the line C-D in Fig. 3e. This cross-section shows clearly that we achieved a resolution of at least $1 \mu \mathrm{m}$, while the illumination wavelength was $\lambda=7.94 \mu \mathrm{m}$. So, we have achieved a resolution at least four times better than the one imposed by the diffraction limit and accessible to the lens-based microscopes. Cell results shown in this article demonstrate that the actual resolution of our SNOM is even higher, but in these $\mathrm{BN}$ samples the distribution of different phases does not allow sharper optical contrast.

\section{SNOM with biological specimens}

After successful tests with material science samples, we found it logical to test the possibilities of this technique with samples most commonly studied with a conventional microscope: cells. In this paper, we show SNOM images of keratinocyte cells (HaCaT cell line). Such cells were seeded on glass cover slides; after $24 \mathrm{~h}$ the growth medium was removed and the cells were fixed in paraformaldehyde and washed twice with phosphate-buffered saline (PBS) and twice with distilled water.

We performed the test scans targeting IR absorptions of several chemical bonds and cell constituents. We used FEL to illuminate the sample at different wavelengths: Fig. $4 \mathrm{~d}$ : $\lambda=6.1 \mu \mathrm{m}$, targeting the so called amide I absorption band (corresponding to the $\mathrm{C}=\mathrm{O}$ bond stretch absorption. The $\mathrm{C}=\mathrm{O}$ bond is present in all the proteins, at every link between two amino-acids); Fig. 4c: $\lambda=6.95 \mu \mathrm{m}$, wavelength strongly absorbed by the sulfide stretch band (mainly from the cell growth medium); Fig. 4b: $\lambda=8.05 \mu \mathrm{m}$, strongly absorbed by the phosphorus stretch band (mainly concentrated in the DNA and the RNA). Fig. 4a shows the corresponding topography.

Darker color in Fig. 4d indicates the position of proteins. Proteins are quite homogeneously distributed in cells and, as expected, there is a steady absorption arising from all over the cell. The cross-section (Fig. 4e) of the reflected signal along the line A-B (Fig. 4d) allows us to asses the resolution of our setup: it is of the order of $100 \mathrm{~nm}$, or $\lambda / 60$. This is at least 30 times better than the diffraction limited lens-based microscopes.

Fig. 4c also shows a steady absorption from all over the cell, indicating that even washed, our cells still contain important amounts of the cell growth medium, while the cover-slip substrate does not.

Fig. $4 \mathrm{~b}$ shows important absorption only on one part of the cell (within the white circle in the figure). This is due to the localization of the DNA inside the nucleus, which yields a strong local absorption seen in the SNOM image. FT-IR measurements show only a relatively low absorption at this wavelength. Such absorption has been seen with FT-IR only on cells during replication, possibly because of the spatial extension increase of the nucleus [21].

\section{Conclusions}

Our experiments demonstrate that IR-SNOM is an extremely powerful analytical instrument. It combines the IR spectroscopy's high chemical specificity with the SNOM's high spatial resolution. The results demonstrated that the resolution of our SNOM setup is around $100 \mathrm{~nm}$, independent of the wavelength. Images showing resolutions of the order of $\lambda / 60$, or at least 30 times better than the diffraction limited lens-based microscopes, have been presented.

Boron nitride $(\mathrm{BN})$ thin film results demonstrate that SNOM has very interesting materials science applications. IR-SNOM shows the distribution of hexagonal and cubic phases within the sample pointing out that close to the silicon substrate there is a layer of h-BN, while the cubic phase (c-BN) appears only farther from it.

The cell results demonstrate that the technique can easily be applied to biological specimens and the resolution achieved suggests that the IR-SNOM can bring a very powerful insight into some of the most important bio-med research topics. 


\section{Acknowledgements}

We thank the entire staff of the W.M. Keck Foundation Free Electron Laser Center at Vanderbilt University for their able assistance.

This work is supported by the Italian National Research Council, Ecole Polytechnique Fédérale de Lausanne, the Fonds National Suisse de la Recherche Scientifique, the National Institutes of Health and the United States Office of Naval Research.

\section{References}

[1] J.A. O'Keefe, J. Opt. Soc. Am. 46 (1956) 359

[2] M. Born, E. Wolf, Principles of Optics, 6th ed., Pergamon Press, Oxford, 1987.

[3] E.A. Ash, G. Nichols, Nature 237 (1972) 510.

[4] D. Vobornik, G. Margaritondo, J.S. Sanghera, P. Thielen, I.D. Aggarwal, B. Ivanov, J.K. Miller, R. Haglund, N.H. Tolk, A. CongiuCastellano, M.A. Rizzo, D.W. Piston, F. Somma, G. Baldacchini, F. Bonfigli, T. Marolo, F. Flora, R.M. Montereali, A. Faenov, T. Pikuz, G. Longo, V. Mussi, R. Generosi, M. Luce, P. Perfetti, A. Cricenti, Infrared Phys. Technol. 45 (2004) 409.

[5] D.W. Pohl, W. Denk, M. Lanz, Appl. Phys. Lett. 44 (1984) 651.

[6] E. Betzig, J.K. Trautman, Science 257 (1992) 189.

[7] E. Betzig, P.L. Finn, J.S. Wiener, Appl. Phys. Lett. 60 (1994) 2484.

[8] H. Heinzelmann, D.W. Pohl, Appl. Phys. A 59 (1994) 89.
[9] D.W. Pohl, D. Courjon (Eds.), NATO ASI Series "Near-Field Optics", vol. 262, Kluwer Academic Press, 1992.

[10] A. Cricenti, R. Generosi, M. Luce, P. Perfetti, G. Margaritondo, D. Talley, J.S. Sanghera, I.D. Aggarwal, N.H. Tolk, A. CongiuCastellano, M.A. Rizzo, D.W. Piston, Biophys. J. 85 (2003) 2705.

[11] A. Cricenti, V. Marocchi, R. Generosi, M. Luce, P. Perfetti, D. Vobornik, G. Margaritondo, D. Talley, P. Thielen, J.S. Sanghera, I.D. Aggarwal, J.K. Miller, N.H. Tolk, D.W. Piston, J. Alloys Compd. 362 (2004) 21.

[12] A. Cricenti, G. Longo, M. Luce, R. Generosi, P. Perfetti, D. Vobornik, G. Margaritondo, P. Thielen, J.S. Sanghera, I.D. Aggarwal, J.K. Miller, N.H. Tolk, D.W. Piston, F. Cattaruzza, A. Flamini, T. Prosperi, A. Mezzi, Surf. Sci. 544 (2003) 51.

[13] B.B. Akhremitchev, S. Pollack, G.C. Walker, Langmuir 17 (2001) 2774.

[14] B.B. Akhremitchev, Y. Sun, L. Stebounova, G.C. Walker, Langmuir 18 (2002) 5325.

[15] D.B. Talley, L.B. Shaw, J.S. Sanghera, I.D. Aggarwal, A. Cricenti, R. Generosi, M. Luce, G. Margaritondo, J.M. Gilligan, N.H. Tolk, Mater. Lett. 42 (2000) 339.

[16] D.T. Schaafsma, Opt. Eng. 38 (1999) 1381.

[17] D.T. Schaafsma, R. Mossadegh, J.S. Sanghera, I.D. Aggarwal, J.M. Gilligan, N.H. Tolk, M. Luce, R. Generosi, P. Perfetti, A. Cricenti, G. Margaritondo, Ultramicroscopy 77 (1999) 77.

[18] B. Dragnea, J. Preusser, W. Schade, S.R. Leone, J. Appl. Phys. 86 (1999) 2795.

[19] B. Dragnea, J. Preusser, J.M. Szarko, L.A. McDonough, S.R. Leone, W.D. Hinsberg, Appl. Surf. Sci. 175-176 (2001) 783.

[20] J. Tian, L. Xia, X. Ma, Y. Sun, E.-s. Byon, S.-h. Lee, S.-r. Lee, Thin Solid Films 355-356 (1999) 229.

[21] M. Diem, S. Boydston-White, L. Chiriboga, Appl. Spectrosc. 53 (1999) 148A. 